About ten variables pertaining to digestive diseases in the piglet and respiratory disorders in the fattening pig were found to have an effect on growth and constitute sanitary indexes. Individual and sanitary growth parameters were characterized by a withinherd component of their total high variance (often ranging between 55 and $65 \mathrm{p} .100$ of the total variance).

Pneumonia lesions observed at the abattoir led to increases in age at $100 \mathrm{~kg}$ ranging between 4 and 13 p. 100 according to their extent, and to decreases in DMG in late fattening (110 to 185 days) ranging between 7 and 19 p. 100 . Pneumonia accounted for more than 6 p. 100 of the total variance of age at $100 \mathrm{~kg}$. Respiratory symptoms (coughing and sneezing) also represented important indexes which have to be taken into account to determine the economic consequences of health problems.

\title{
Experimental pathogenicity of Pasteurella multocida and effect of the exotoxin in the piglet
}

\author{
Marylène KOBISCH
}

Station de Pathologie Porcine, B.P. 9, 22440 Ploufragan

A total of 28 S.P.F. piglets were intranasally and intratracheally infected with $P$. multocida (type A or D) or were administered by the intramuscular or intraperitoneal route the crude dermonecrotic exotoxin of these bacteria. Ten S.P.F. piglets received the culture medium in the same conditions.

The experimental infection provoked the death of some piglets and led to severe lesions of the respiratory tract and organs but also of the liver and urinary tract. Results showed that $P$. multocida type A is related to the occurrence of pneumonia, whereas $P$. multocida type $\mathrm{D}$ is rather associated with nasal lesions.

\section{Streptococcal diseases in the pig}

\author{
Josée VAISSAIRE, Micheline LAROCHE \\ Laboratoire Central de Recherches Vétérinaires, Service de Bactériologie, \\ 22, rue Pierre-Curie, 94700 Maisons-Alfort
}

The authors give an overview of streptococcal diseases in the pig over the last six years in France. A total of 4152 strains of streptococci were isolated from 877 outbreaks of porcine streptococcal diseases mainly in industrial farms.

The different strains were studied by chemical characterization and serogrouping by the Lancefield or the Fuller method. Clinical observation and symptomatology were usually typical for the affections due to the same serogroup. Theses affections can also be zoonoses.

Results emphasized the predominance of affections due to Streptococcus suis (groups R and S) and to Str. equisimilis (group C) : 19 p. 100.

Affections caused by serotypes $\mathrm{E}, \mathrm{L}$ and $\mathrm{G}$ and causing abortions and mortinatality represented 9 p. 100 of the cases. Rapidly evoluting septicaemia were observed in 8 p. 100 of the cases among adults or fattening pigs and were due to Str. agalactiae, dysgalactiae and uberis. Enterococci were responsible for septicaemia (6 p. 100 of the cases). Finally, 10 p. 100 of the affections were due to nongroupable streptococci (Str. mitis, sanguis). These affections may cause important economic losses. Their treatment is difficult and their prevention remains essential. 